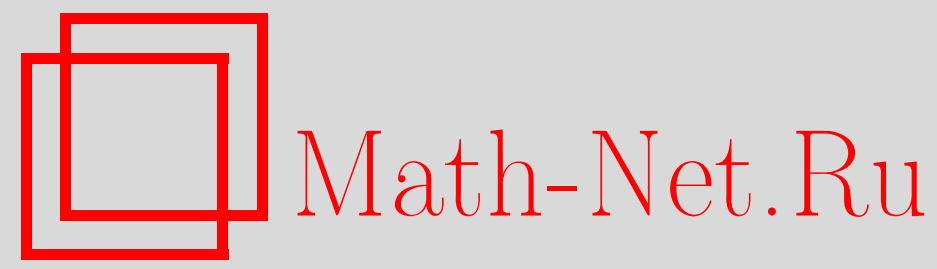

С. Я. Шоргин, О точности нормальной аппроксимации распределений случайных сумм с безгранично делимыми индексами, Теория вероятн. и ее примен., 1996, том 41, выпуск 4, 920-926

DOI: https://doi.org/10.4213/tvp3283

Использование Общероссийского математического портала Math-Net.Ru подразумевает, что вы прочитали и согласны с пользовательским соглашением http://www . mathnet.ru/rus/agreement

Параметры загрузки:

IP : 54.210 .77 .194

26 апреля 2023 г., 14:31:25






\section{О ТОЧНОСТИ НОРМАЛЬНОЙ АППРОКСИМАЦИИ РАСПРЕДЕЛЕНИЙ СЛУЧАЙНЫХ СУММ С БЕЗГРАНИЧНО ДЕЛИМЫМИ ИНДЕКСАМИ}

Рассматриваются случайные суммы независимых одинаково распределенных случайных величин с индексом, имеющим безгранично делимое (и тем самым обобщенное пуассоновское) распределение. Получены оценки точности нормальной аппроксимапки распределений указанных случайных сумм; в правых частях оценок присутствуют «нецентральные» ляпуновские дробн. Обсуждается вопрос о соотношении величин «классических》 и «неентральных》 ляпуновских дробей; показано, что в рамках данной задачи использование последних более естественно.

Ключевые слова и Фразы: случайные суммы, индекс и случайные слагаемые случайной суммы, «нецентральное» и «классическое» отношения Ляпунова, безгранично делимое распределение, пуассоновское и обобщенное пуассоновское распределения, нормальная аппроксимация.

1. Основные обозначения и проблематика работы. Введем, прежде всего, ряд обозначений, упрощающих запись результатов и доказательств.

Совпадение распределений случайных величин (с.в.) $X$ и $Y$ будем обозначать как $X={ }_{d} Y$. Функцию распределения и характеристическую функцию (х.ф.) любой с.в. $X$ обозначим $F_{X}(x)$ и $f_{X}(t)$, соответственно, а производящую функцию (п.ф.) любой неотрицательной целочисленной (н.ц.) с.в. $N$ обозначим $\psi_{N}(z)$.

Пусть $N$ - некоторая н.ц. с.в., $X$ - произвольная с.в. Обозначим случайную величину, х.ф. распределения которой равна $\psi_{N}\left(f_{X}(t)\right)$, символом $\{N, X\}$. Очевидно, что с.в. $\{N, X\}$ может быть представлена в виде $\{N, X\}={ }_{d} \sum_{j=1}^{N} X_{j}$ (для определенности полагаем, что $\sum_{j=1}^{0}=0$ ), где $X_{1}, X_{2}, \ldots$ - одинаково распределенные (о.р.) с.в., причем $X_{j}={ }_{d} X$ и с.в. $N, X_{1}, X_{2}, \ldots$ независимы в совокупности. Будем называть при этом с.в. $\{N, X\}$ случайной суммой (сл. суммой), с.в. $N$ - индексом, а с.в. $X$ - случайным слагаемым (сл. слаг.). Очевидно, что в случае, когда $X$ - н.ц. с.в., то и $\{N, X\}$ - н.ц. с.в., и ее п.ф. $\psi_{\{N, X\}}(z)=\psi_{N}\left(\psi_{X}(z)\right)$.

Для любой с.в. $X$, имеющей два. конечных первых момента, символом $\widetilde{X}$ или $X^{-}$ обозначим нормированную с.в.: $\widetilde{X}=_{d}(X-\mathbf{E}(X)) /[\mathbf{D}(X)]^{1 / 2}$.

Если невырожденная в 0 с.в. $X$ имеет три конечных первых момента, то назовем отношением Ляпунова величину $L(X)=\mathbf{E}\left(|X|^{3}\right) /\left[\mathbf{E}\left(X^{2}\right)\right]^{3 / 2}$. При $\mathbf{E}(X)=0$ эта величина совпадает с «классической» ляпуновской дробью $L(X-\mathbf{E}(X))$, фигурирующей в правой части оценки Берри-Эссеена (й содержащей соответствующие центральные моменты). Для любой невырожденной с.в. $X$, имеюшей три момента, обозначим $L_{0}(X)=L(X-\mathbf{E}(X))$.

Предположим, что распределение с.в. $N$ - безгранично делимое (б.д.) в классе распределений н.ц. с.в., т.е. для любого натурального $m$ существует такая н.ц. с.в. $N_{m}^{\prime}$, что $N={ }_{d}\left\{m, N_{m}^{\prime}\right\}$. Как известно [1], в этом случае распределение с.в. $N$ является обобщенным пуассоновским (о.п.), т.е. ее х.ф. равна $f_{N}(t)=\exp \left[\lambda\left(f_{Y}(t)-1\right)\right]$, где $\lambda>0, f_{Y}(t)$ - х.ф. некоторой н.ц. с.в. $Y$; иначе говоря, $N={ }_{d}\{\Pi(\lambda), Y\}$, где $\Pi(\lambda)$ - пуассоновская с.в. с параметром $\lambda$. Пусть существуют первые три момента с.в. $N$ и $X$.

\footnotetext{
*Институт проблем информатики РАН, ул. Вавилова, 30/6, 117900 Москва, Рос-
} сия. 
Предметом работы является оценка точности аппроксимации распределения с.в. $S={ }_{d}\{N, X\}$ в случае, когда с.в. $N$ имеет б.д. распределение, нормальным законом с соответствующими моментами. Очевидно, что в этом случае $S={ }_{d}\{\{\Pi(\lambda), Y\}, X\}$. Положим $\Delta=\sup _{x}\left|F_{\widetilde{S}}(x)-\Phi(x)\right|$, где $\Phi(x)$ - стандартная нормальная функция распределения.

Для случая произвольного распределения индекса $N$ существует ряд ощенок точности нормальной аппроксимации распределений сл. сумм $S={ }_{d}\{N, X\}$. Оценки из [2] для случая $\mathbf{E}(X)=0$ представляются близкими к окончательным. Что касается общего случая $(\mathbf{E}(X) \neq 0)$, то соответствуюцие оценки точности нормальной аппроксимации [3], [4] весьма сложны по структуре; при этом в указанщые оценки входит компонента, содержащая «классическое» отношение Ляпунова $L_{0}(X)$ ([3, теорема 6.2.1]; см. ниже неравенство (6)). В то же время анализ случая; когда с.в. $N$ имеет распределение Пуассона (частный случай рассматриваемой нами задачи), показывает, что при $\mathbf{E}(X) \neq 0$ более естественным является наличие в оценках для $\Delta \ll н е-$ центральных» ляпуновских дробей вида $L(X)$. Оценку величины $\Delta$ для $N={ }_{d} \Pi(\lambda)$ в терминах величин $L(X)$ сформулируем в качестве леммы.

Лемма 1. Если $N$ имеет распределение Пуассона с параметром $\lambda$, то

$$
\Delta \leqslant C_{1} \frac{L(X)}{\lambda^{1 / 2}}
$$

где $C_{1}$ - абсолютная постоянная.

Отметим, что данная оценка, по-видимому, впервые приведена в [5] (без доказательства; при этом утверждалось, что можно положить $C_{1}=2.23$ ). В [6] приводится доказательство цанной леммы; показано, что можно положить $C_{1}=1.99$. Наконец, недавно совместно В. Ю. Королевым и автором показано, что можно положить $C_{1}=C_{0}$, где $C_{0}$ - постоянная из неравенства Берри-Эссеена для одинаково распределенных слагаемых, т.е. (см. [7]) $C_{1}=C_{0}=0.7655$ (доказательство последней оценки для $C_{1}$ будет опубликовано позднее).

Основной результат работы - неравенство (2), являющееся обобщением (1) на случай о.п. распределения индекса $N$. Эта оценка является альтернативной результатам [3], [4] (хотя и относится к более узкому классу распределений, чем рассматривается в этих работах). В случае $N={ }_{d} \Pi(\lambda)$ ощенка (2) сводится к (1); причем абсолютная постоянная в (2) совпадает с $C_{1}$ из (1).

При решении задачи оценки точности аппроксимации распределения сл. сумм с о.п. распределением индекса, безусловно, имеет место существенная потеря общности по сравнению с ситуацией произвольного распределения $N$, рассмотренной, например, в [2]-[4]. Но все же и данный частный случай представляет достаточный интерес. Так, целочисленный случайный процесс с независимыми приращениями представляет собой совокупность с.в. $N_{t}$, имеющих б.д. распределение (и; значит, о.п. распределение [1]); задачи исследования сл. сумм, связанных с такими процессами, возникают при анализе ряда систем массового обслуживания, процессов риска в страховой математике и т.п. Отметим при этом, что оценки, получаемые в данной работе, оказываются весьма простыми по форме и (см. п. 3) в ряде ситуаций более точны, чем общие оценки [3], [4].

2. Формулировка и обсуждение результатов. Пусть с.в. $N$ и $X$ имеют три конечных первых момента.

Теорема 1. Справедлива оченка

$$
\begin{aligned}
\Delta \leqslant & \frac{C_{1}}{\lambda^{1 / 2}}\left\{\mathbf{E}[Y(Y-1)(Y-2)] \mathbf{E}^{3}(|X|)+3 \mathbf{E}[Y(Y-1)] \mathbf{E}(|X|) \mathbf{E}\left(X^{2}\right)\right. \\
& \left.+\mathbf{E}(Y) \mathbf{E}\left(|X|^{3}\right)\right\} /\left\{\mathbf{E}\left(Y^{2}\right) \mathbf{E}^{2}(X)+\mathbf{E}(Y) \mathbf{D}(X)\right\}^{3 / 2}
\end{aligned}
$$

аде $C_{1}-$ абсолютяая постоннная из неравенства (1). 
(Отметим, что для н.ц. с.в. $Y$ справедливы неравенства $\mathbf{E}[Y(Y-1)(Y-2)] \geqslant 0$, $\mathbf{E}[Y(Y-1)] \geqslant 0$.

Неравенство (2) является самым точным из результатов работы, но имеет достаточно сложный вид. Более наглядным результатом является следующее утверждение.

Следствие 1. Справедлива оценка

$$
\begin{aligned}
\Delta & \leqslant \frac{C_{1}}{\lambda^{1 / 2}} \frac{\mathbf{E}\left(Y^{3}\right) \mathbf{E}\left(|X|^{3}\right)}{\left\{\mathbf{E}\left(Y^{2}\right) \mathbf{E}^{2}(X)+\mathbf{E}(Y) \mathbf{D}(X)\right\}^{3 / 2}} \\
& =\frac{C_{1}}{\lambda^{1 / 2}} L(X) L(Y)\left[\frac{\mathbf{E}\left(X^{2}\right)}{\mathbf{E}^{2}(X)+\mathbf{E}(Y) \mathbf{D}(X) / \mathbf{E}\left(Y^{2}\right)}\right]^{3 / 2} .
\end{aligned}
$$

(Неравенство (3) вытекает из (2) в силу того, что $\mathbf{E}^{3}(|X|) \leqslant \mathbf{E}\left(|X|^{3}\right), \mathbf{E}(|X|) \times$ $\mathbf{E}\left(X^{2}\right) \leqslant \mathbf{E}\left(|X|^{3}\right)$ и $\left.Y(Y-1)(Y-2)+3 Y(Y-1)+Y=Y^{3}.\right)$

Правая часть (3) совпадает с правой частью (2) в случае, когда либо с.в. $X$ является вырожденной, либо с.в. $Y$ вырождена в 1. Очевидно, что в остальных случаях неравенство (3) «хуже», чем (2). Пусть $L_{0}^{\prime}(N)=\mathbf{E}\left[(N-\mathbf{E}(N))^{3}\right] / \mathbf{D}^{3 / 2}(N)$. Так как $\mathbf{E}\left[(\{\Pi(\lambda), Y-\mathbf{E}(Y)\})^{3}\right]=\lambda \mathbf{E}\left(Y^{3}\right)$, то $L_{0}^{\prime}(N)=L(Y) / \lambda^{1 / 2}$.

Неравенство (3) может быть переписано эквивалентным образом с использованием только моментов с.в. $N$ и $X$ (т.е. есть для вычисления значения оценки необязательно знать величину $\lambda$ и моменты с.в. $Y$ ).

Следствие 2. Справедлива оценка

$$
\begin{aligned}
\Delta & \leqslant C_{1} \frac{\mathbf{E}\left[(N-\mathbf{E}(N))^{3}\right] \mathbf{E}\left(|X|^{3}\right)}{\left\{\mathbf{D}(N) \mathbf{E}^{2}(X)+\mathbf{E}(N) \mathbf{D}(X)\right\}^{3 / 2}} \\
& =C_{1} L(X) L_{0}^{\prime}(N)\left[\frac{\mathbf{E}\left(X^{2}\right)}{\mathbf{E}^{2}(X)+\left(\mathbf{E}(N) / \mathbf{E}\left(N^{2}\right)\right) \mathbf{D}(X)}\right]^{3 / 2} .
\end{aligned}
$$

(Данное следствие вытекает из (3) и свойств моментов с.в. $N$.)

Для выражения $\mathbf{E}\left(Y^{2}\right) \mathbf{E}^{2}(X)+\mathbf{E}(Y) \mathbf{D}(X)$, присутствующего в знаменателе правой части неравенства (3), можно выписать следующие нижние оценки: $\mathbf{E}\left(Y^{2}\right) \times$ $\mathbf{E}^{2}(X)+\mathbf{E}(Y) \mathbf{D}(X) \geqslant \mathbf{E}\left(Y^{2}\right) \mathbf{E}^{2}(X)$ и $\mathbf{E}\left(Y^{2}\right) \mathbf{E}^{2}(X)+\mathbf{E}(Y) \mathbf{D}(X) \geqslant \mathbf{E}(Y) \mathbf{E}\left(X^{2}\right)$ (так как $\left.\mathbf{E}\left(Y^{2}\right) \geqslant \mathbf{E}(Y)\right)$. Значит, из (3) вытекает следуюший результат.

Следствие 3. Справедлива оченка

$$
\Delta \leqslant \frac{C_{1}}{\lambda^{1 / 2}} \min \left\{L(Y) \frac{\mathbf{E}\left(|X|^{3}\right)}{\left|\mathbf{E}^{3}(X)\right|}, \frac{\mathbf{E}\left(Y^{3}\right)}{\mathbf{E}^{3 / 2}(Y)} L(X)\right\} .
$$

Очевидно, что данное неравенство (как и все приведенные выше) является обобщением (1) (с сохранением абсолютной постоянной); для получения из теоремы 1 и следствий 1-3 оценки (1) достаточно положить в них $Y={ }_{d} 1$ или, что то же самое, $N={ }_{d} \Pi(\lambda)$.

Отметим, что в случае, когда с.в. $X$ фиксирована, причем $\mathbf{E}(X) \neq 0$, необходимым и достаточным условием сходимости правой части (4) к 0 является $L_{0}^{\prime}(N) \rightarrow 0$. В терминах моментов с.в. $Y$ это условие выглядит так:

$$
\frac{L(Y)}{\lambda^{1 / 2}} \rightarrow 0
$$

Очевидно, что данное условие выполняется, если $\lambda \rightarrow \infty$, а с.в. $Y$, являюшаяся сл. слаг. для сл. суммы $N$, фиксирована. Однако при рассмотрении произвольной последовательности о.п. с.в. $N_{k}={ }_{d}\left\{\Pi\left(\lambda_{k}\right), Y_{k}\right\}$ даже с неограниченно растущим параметром $\lambda_{k}$, но с различными распределениями с.в. $Y_{k}$, выполнение (5) не гарантировано. 
3. Сравнение результатов работы с имеющимися оценками. Ниже осупествляется сравкение оценок, приведенных в предыдущем пункте, с аналогичными оценками, полученными с помощью других методов в [3], [4]. Это сравнение проводится в предположении, что $\lambda \rightarrow \infty$.

В [3] доказано следующее утверждение, уточняющее оценку из [4]: если с.в. $X$ и $N$ имеют три конечных момента, то для любого $q \in(0,1)$

$$
\Delta \leqslant C_{0} \frac{L_{0}(X)}{q^{1 / 2} \mathbf{E}^{1 / 2}(N)}+w(q) \frac{\mathbf{E}(|N-\mathbf{E}(N)|)}{\mathbf{E}(N)}+\sup _{x}\left|F_{\tilde{N}}(x)-\Phi(x)\right|,
$$

где $C_{0}$ - постоянная из оценки Берри-Эссеена для одинаково распределенных слагаемых (см. п. 1),

$$
w(q)=\max \left[\frac{1}{1-q}, \frac{1}{c q^{1 / 2}\left(1+q^{1 / 2}\right)}\right], \quad c=(2 \pi e)^{1 / 2} .
$$

Для того, чтобы сравнить неравенство (6) с результатами из п. 2, следует выяснить, как выглядит (6) в ситуации, когда с.в. $N$ имеет о.п. распределение. Для этого нужно, прежде всего, заменить в правой части (6) величину $\sup _{x}\left|F_{\tilde{N}}(x)-\Phi(x)\right|$ на ее оценку, получаемую с помощью методов [3]-[4]; такая оценка получена в [6]:

$$
\sup _{x}\left|F_{\bar{N}}(x)-\Phi(x)\right| \leqslant \lambda^{-1 / 2}\left[C_{0}+\inf _{0<p<1}\left\{\frac{C_{0} L_{0}(Y)}{p^{1 / 2}}+w(p)\right\}\right] .
$$

Кроме того, в случае, когпа $N={ }_{d}\{\Pi(\lambda), Y\}$, в силу неравенства Ляпунова $\mathbf{E}(|N-\mathbf{E}(N)|) \leqslant \mathrm{D}^{1 / 2}(N)=\lambda^{1 / 2} \mathbf{E}^{1 / 2}\left(Y^{2}\right)$. В результате заключаем, что для с.в. $N$, имеющих о.п. распределение,

$$
\begin{aligned}
\Delta \leqslant \lambda^{-1 / 2}\left\{\inf _{0<q<1}\left\{C_{0} \frac{L_{0}(X)}{q^{1 / 2} \mathbf{E}^{1 / 2}(Y)}+w(q) \frac{\mathbf{E}^{1 / 2}\left(Y^{2}\right)}{\mathbf{E}(Y)}\right\}\right. \\
\left.+C_{0}+\inf _{0<p<1}\left\{\frac{C_{0} L_{0}(Y)}{p^{1 / 2}}+w(p)\right\}\right\} .
\end{aligned}
$$

В силу [6, лемма 3] данное неравенство можно переписать так:

$$
\Delta \leqslant \lambda^{-1 / 2}\left\{\frac{\mathbf{E}^{1 / 2}\left(Y^{2}\right)}{\mathbf{E}(Y)} \Omega\left(r_{1}\right)+C_{0}+\Omega\left(r_{2}\right)\right\}
$$

где

$$
\Omega(r)=\left\{\begin{array}{lll}
\frac{1+s^{2}(r / 2)}{\left(1-s^{2}(r / 2)\right)^{2}} & \text { при } & r \geqslant \frac{2(1+c)}{\left(2 c+c^{2}\right)^{2}} \\
r(1+c)+\frac{(1+c)^{2}}{2 c+c^{2}} & \text { при } & r \leqslant \frac{2(1+c)}{\left(2 c+c^{2}\right)^{2}}
\end{array}\right.
$$

$s(u)$ - решение уравнения $s^{3}(u) /\left(1-s^{2}(u)\right)^{2}=u$ (которое существует и единственно при любом $u>0) ; r_{1}=C_{0} \sqrt{\mathbf{E}(Y) / \mathbf{E}\left(Y^{2}\right)} L(X) ; r_{2}=C_{0} L_{0}(Y)$. Отметим, что $r_{2} \geqslant$ $2(1+c) /\left(2 c+c^{2}\right)^{2}$.

Точное решение вопроса, в каких случаях лучше оценка из (2), а в каких - оценка из (7), представляется затруднительным в силу сложности выражений, стоящих в правых частях этих неравенств. Поэтому для практического применения можно рекомендовать «объединенную» оценку:

$$
\Delta \leqslant \min \{P, Q\}
$$

где $P$ - правая часть неравенства (2), $Q-$ правая часть (7). 
Для корректной постановки задачи сравнения асимптотического поведения этих оценок при $\lambda \rightarrow \infty$ рассмотрим следуюшгую схему серий. Пусть при каждом $n$ заданы положительный параметр $\lambda_{n}$, с.в. $M_{n}$, имеющая пуассоновское распределение с параметром $\lambda_{n}$, последовательность о.р. н.щ. с.в. $Y_{1 n}, Y_{2 n}, \ldots$ и последовательность о.p. с.в. $X_{1 n}, X_{2 n}, \ldots$, причем при хаждом $n$ все перечисленные с.в. независимы в совокупности. При различных $n$ как с.в. $Y_{i n}$, так и с.в. $X_{i n}$ могут быть распределены по-разному. Рассмотрим последовательности сл. сумм $N_{n}={ }_{d} Y_{1 n}+\cdots+Y_{M_{n} n}$, $S_{n}={ }_{d} X_{1 n}+\cdots+X_{M_{n} n}$ и последовательность величин $\Delta_{n}=\sup _{x}\left|F_{\bar{S}_{n}}(x)-\Phi(x)\right|$.

Пусть с.в. $Y_{n}={ }_{d} Y_{1 n}, X_{n}={ }_{d} X_{1 n}$. Тогда $N_{n}={ }_{d}\left\{\Pi\left(\lambda_{n}\right), Y_{n}\right\}$ и $S_{n}={ }_{d}\left\{N_{n}, X_{n}\right\}$. Отметим, что значение $\Delta_{n}$ определяется тройкой $\left(\lambda_{n}, Y_{n}, X_{n}\right)$ (естественно, имеются в виду распределения с.в. $Y_{n}$ и $\left.X_{n}\right)$.

Итак, справедливы неравенства $\Delta_{n} \leqslant P_{n}, \Delta_{n} \leqslant Q_{n}$, где $P_{n}$ - правая часть неравенства (2) для $\lambda=\lambda_{n}, Y={ }_{d} Y_{n}, X={ }_{d} X_{n} ; Q_{n}$ - правая часть неравенства (7) при этих же $\lambda, Y, X$. Конечно, значения $P_{n}$ и $Q_{n}$ также зависят от тройки $\left(\lambda_{n}, Y_{n}, X_{n}\right)$, поэтому при необходимости будет использоваться запись $P_{n}\left(\lambda_{n}, Y_{n}, X_{n}\right)$ и $Q_{n}\left(\lambda_{n}, Y_{n}, X_{n}\right)$.

Следующая теорема показывает, что существует некоторое естественное множество распределений с.в. $Y_{n}$ (см. условие (8)), такое, что для всех с.в. $X_{n}$, имеющих три момента, и для $Y_{n}$, удовлетворяющих (8), оценка $P_{n}$ в определенном смысле более точна, чем оценка $Q_{n}$.

Теорема 2. Рассмотрим с.в. $Y_{n}$, удовлетворяюиие условию

$$
\mathbf{E}\left(Y_{n}^{3}\right) \mathbf{E}^{1 / 2}\left(Y_{n}\right) \leqslant K \mathbf{E}^{3 / 2}\left(Y_{n}^{2}\right),
$$

где $K \geqslant 1$ есть абсолютная постояняая. Существует абсолютная постоянная $R$ (зависячая от $K$ ) такая, что для любых $\lambda_{n}>0$, любых с.в. $Y_{n}$, удовлетворяючих (8), и мюбых с.в. $X_{n}$, имеющих три конечных момента, $P_{n} / Q_{n} \leqslant R$. $B$ то же время существует такая последовательность троек $\left(\lambda_{n}^{0}, Y_{n}^{0}, X_{n}^{0}\right)$, где с.в. $Y_{n}^{0}$ удовлетворяют (8), а с.в. $X_{n}^{0}$ имеют три конечных момента, что $P_{n}\left(\lambda_{n}^{0}, Y_{n}^{0}, X_{n}^{0}\right) \rightarrow 0$ при $\lambda_{n} \rightarrow \infty$, но $Q_{n}\left(\lambda_{n}^{0}, Y_{n}^{0}, X_{n}^{0}\right)$ не стремится $\propto 0$.

3 а м е ч а н и е 1. Из этой теоремы следует, что (в рамках описанного класса распределений с.в. $Y_{n}$ и $X_{n}$ ) для всех тех последовательностей распределений с.в. $S_{n}$, асимптотическую нормальность которых гарантирует оценка $Q_{n}$, оценка $P_{n}$ также сообщает об их асимптотической нормальности (и имеет «не худший » порядок скорости сходимости х 0 , чем $P_{n}$ ). В то же время наличие такой последовательности троек $\left(\lambda_{n}^{0}, Y_{n}^{0}, X_{n}^{0}\right)$, что оценка $P_{n}$ «улавливает» асимптотическую нормальность соответствующей последовательности распределений с.в. $S_{n}$, а оценка $Q_{n}$ этого не «улавливает», означает, что в рамках данного класса распределений с.в. $Y_{n}$ и $X_{n}$ оценка вида $P_{n}$ является асимптотически более точной, чем оценка вида $Q_{n}$. Естественно, можно выделить и такой класс распределений с.в. $Y_{n}$ и $X_{n}$, в рамках которого асимптотически более точной является оценка $Q_{n}$, однако в данной работе этот вопрос не рассматривается.

3 а м е ч а н и е 2. Отметим, что условиям (8) удовлетворяют, в частности, с.в. $Y_{n}$, удовлетворяющие условию $\mathrm{E}\left(Y_{n}^{3}\right) \leqslant K \mathrm{E}\left(Y_{n}^{2}\right)(K-$ абсолютная постоянная). Это следует из того, что $\mathrm{E}\left(Y_{n}^{3}\right) \mathbf{E}^{1 / 2}\left(Y_{n}\right) \leqslant K \mathrm{E}\left(Y_{n}^{2}\right) \mathrm{E}^{1 / 2}\left(Y_{n}\right) \leqslant K \mathbf{E}^{3 / 2}\left(Y_{n}^{2}\right)$. В частности, этому условию удовлетворяют все с.в., равномерно ограниченные величиной $K$.

3 а м е ч н и е 3 . Неравенство $K \geqslant 1$ вытекает из (10).

4. Доказательство результатов работы. Важнейшее значение для доказательства теоремы 1 имеет следующая простая лемма.

Лемма 2. Если $Y$ - н.ч. с.в., то

$$
S={ }_{d}\{\{\Pi(\lambda), Y\}, X\}={ }_{d}\{\Pi(\lambda),\{Y, X\}\} .
$$


Д о к а з а т ль с т в о сводится к цепочке тождеств:

$$
\begin{aligned}
f_{S}(t) & =\psi_{\{\Pi(\lambda), Y\}}\left(f_{X}(t)\right)=\psi_{\Pi(\lambda)}\left(\psi_{Y}\left(f_{X}(t)\right)\right) \\
& =\psi_{\Pi(\lambda)}\left(f_{\{Y, X\}}(t)\right)=f_{\{\Pi(\lambda),\{Y, X\}\}}(t) .
\end{aligned}
$$

Из леммы 2 следует, что с.в. $S={ }_{d}\{\{\Pi(\lambda), Y\}, X\}$, являющаяся сл. суммой с обобщенным пуассоновским индексом $\{\Pi(\lambda), Y\}$ и сл. слаг. $X$, одновременно является сл. суммой с пуассоновским индексом $\Pi(\lambda)$ и сл. слаг. $\{Y, X\}$; благодаря этому анализ распределения с.в. $S$ можно провести с помощью результатов для сл. сумм с пуассоновским индексом.

Д ок а з а те л ь с т в о т е о р е мы 1 . В силу леммы 2 имеем: $S={ }_{d}\{\Pi(\lambda)$, $\{Y, X\}\}$. Пусть $U={ }_{d}\{Y, X\}$. Из леммы 1 следует, что

$$
\left|F_{\widetilde{S}}(x)-\Phi(x)\right|=\left|F_{\{\Pi(\lambda), U\}^{-}}-\Phi(x)\right| \leqslant C_{1} \frac{\mathbf{E}\left(|U|^{3}\right)}{\lambda^{1 / 2} \mathbf{E}^{3 / 2}\left(U^{2}\right)} .
$$

Очевидно, что

$$
\begin{aligned}
\mathbf{E}\left(|U|^{3}\right) \leqslant & \mathbf{E}\left(|\{Y,|X|\}|^{3}\right)=\left[\mathbf{E}\left(Y^{3}\right)-3 \mathbf{E}\left(Y^{2}\right)+2 \mathbf{E}(Y)\right] \mathbf{E}^{3}(|X|) \\
& +3\left[\mathbf{E}\left(Y^{2}\right)-\mathbf{E}(Y)\right] \mathbf{E}(|X|) \mathbf{E}\left(X^{2}\right)+\mathbf{E}(Y) \mathbf{E}\left(|X|^{3}\right) \\
\mathbf{E}\left(U^{2}\right)= & {\left[\mathbf{E}\left(Y^{2}\right)-\mathbf{E}(Y)\right] \mathbf{E}^{2}(X)+\mathbf{E}(Y) \mathbf{E}\left(X^{2}\right) }
\end{aligned}
$$

откуда и следует утверждение теоремы 1.

Доказательство теоремы 2 основывается на следующем утверждении.

Лемма 3. Для любой невырождеяной с.в. $Z$, имеючей три кояечяых момента, $L(Z) \leqslant C_{2} L_{0}(Z)$, причем можко положить $C_{2}=2 \sqrt{2}<2.83$.

Д ок аз ате льс т в о. Пусть $\mathrm{E}(Z)=\alpha, \mathrm{D}(Z)=\sigma^{2}, Z_{0}=Z-\alpha$; не ограничивая общности, предположим, что $\alpha>0$. Тогда

$$
\begin{aligned}
L(Z) & =\frac{\mathbf{E}\left(\left|Z_{0}+\alpha\right|^{3}\right)}{\left(\sigma^{2}+\alpha^{2}\right)^{3 / 2}} \leqslant \frac{\mathbf{E}\left(\left|Z_{0}\right|^{3}\right)+3 \sigma^{2} \alpha+3 \mathbf{E}\left(\left|Z_{0}\right|\right) \alpha^{2}+\alpha^{3}}{\left(\sigma^{2}+\alpha^{2}\right)^{3 / 2}} \\
& \leqslant \mathbf{E}\left(\left|Z_{0}\right|^{3}\right) \frac{\sigma^{3}+3 \sigma^{2} \alpha+3 \sigma \alpha^{2}+\alpha^{3}}{\sigma^{3}\left(\sigma^{2}+\alpha^{2}\right)^{3 / 2}} .
\end{aligned}
$$

Поскольку $L_{0}(Z)=\mathbf{E}\left(\left|Z_{0}\right|^{3}\right) / \sigma^{3}$, то $L(Z) \leqslant L_{0}(Z) \max _{x} \geqslant 0\left[(1+x)^{3} /\left(1+x^{2}\right)^{3 / 2}\right]$.

Максимум в правой части последнего неравенства достигается при $x=1$ и равен $2 \sqrt{2}$, откуда и следует утверждение леммы.

Д ок а з а т ел ь с т в о т е о р е м ы 2. Во-первых, докажем, что существует такая абсолютная постоянная $R$, что $P_{n} / Q_{n} \leqslant R$ для любой последовательности $\left(\lambda_{n}, Y_{n}, X_{n}\right)$, где $Y_{n}$ удовлетворяют (8), а $X_{n}$ имеют три момента. Отметим, что при $r \leqslant 2(1+c) /\left(2 \dot{c}+c^{2}\right)^{2}$ выполняется $\Omega(r) \geqslant R_{1} r$, где $R_{1}=1+c+(1+c)\left(2 c+c^{2}\right) / 2$. Далее, при $r \geqslant 2(1+c)\left(2 c+c^{2}\right)^{2}$

$$
\frac{\Omega(r)}{r}=\frac{1+s^{2}(r / 2)}{2\left(1-s^{2}(r / 2)\right)^{2}(r / 2)}=\frac{1+s^{2}(r / 2)}{2 s^{3}(r / 2)} \text {. }
$$

Последняя дробь - монотонно убывающая функция от $r$; значит,

$$
\frac{\Omega(r)}{r} \geqslant R_{2}=\frac{1+s^{2}\left((1+c) /\left(2 c+c^{2}\right)^{2}\right)}{2 s^{3}\left((1+c) /\left(2 c+c^{2}\right)^{2}\right)} .
$$

При $r \geqslant 0$ выполняется $\Omega(r) \geqslant R_{3} r$, где $R_{3}=\min \left\{R_{1}, R_{2}\right\}$. Имеем:

$$
\lambda_{n}^{1 / 2} Q_{n}>\frac{\mathbf{E}^{1 / 2}\left(Y_{n}^{2}\right)}{\mathbf{E}\left(Y_{n}\right)} \Omega\left(r_{1}\right) \geqslant \frac{C_{0} R_{3}}{\mathbf{E}^{1 / 2}\left(Y_{n}\right)} L_{0}\left(X_{n}\right) .
$$


Рассмотрим теперь оценку $P_{n}$, которую мы возьмем из следствия 1 :

$$
\lambda_{n}^{1 / 2} P_{n}=C_{1} L\left(X_{n}\right) L\left(Y_{n}\right)\left[\frac{\mathbf{E}\left(X_{n}^{2}\right)}{\mathbf{E}^{2}\left(X_{n}\right)+\mathbf{E}\left(Y_{n}\right) \mathbf{D}\left(X_{n}\right) / \mathbf{E}\left(Y_{n}^{2}\right)}\right]^{3 / 2} .
$$

Из неравенства Ляпунова $\mathbf{E}^{2}\left(Y_{n}^{2}\right) \leqslant \mathbf{E}\left(Y_{n}^{3}\right) \mathbf{E}\left(Y_{n}\right)$ и (8) следует, что

$$
1 \leqslant \frac{\mathbf{E}^{1 / 2}\left(Y_{n}^{2}\right)}{\mathbf{E}^{1 / 2}\left(Y_{n}\right)} \leqslant \frac{\mathbf{E}\left(Y_{n}^{3}\right)}{\mathbf{E}^{3 / 2}\left(Y_{n}^{2}\right)} \mathbf{E}^{1 / 2}\left(Y_{n}\right) \leqslant K .
$$

Значит,

$$
\lambda_{n}^{1 / 2} P_{n} \leqslant \frac{K C_{1} L\left(X_{n}\right)}{\mathbf{E}^{1 / 2}\left(Y_{n}\right)}\left[\frac{\mathbf{E}^{2}\left(X_{n}\right)+\mathrm{D}\left(X_{n}\right)}{\mathbf{E}^{2}\left(X_{n}\right)+K^{-2} \mathrm{D}\left(X_{n}\right)}\right]^{3 / 2} \leqslant \frac{C_{1} K^{4} L\left(X_{n}\right)}{\mathbf{E}^{1 / 2}\left(Y_{n}\right)}
$$

Итак, $P_{n} / Q_{n} \leqslant\left(C_{1} K^{4} /\left(C_{0} R_{3}\right)\right) L(X) / L_{0}(X) ;$ в силу леммы 3

$$
\frac{P_{n}}{Q_{n}} \leqslant R=\frac{C_{1} C_{2} K^{4}}{C_{0} R_{3}}
$$

Первое из утверждений теоремы 2 доказано. Для доказательства второго утверждения рассмотрим $\lambda_{n}^{0}=n$, с.в. $Y_{n}^{0}={ }_{d} 1$ и с.в. $X_{n}^{0}$, принимающие два значения: 2 с вероятностью $1 / n$ и 1 с вероятностью $1-1 / n, n=2,3, \ldots$; тогда при всех $n \geqslant 2$ справедливы оценки $L_{0}\left(X_{n}^{0}\right)>0.68 n^{1 / 2}$ и $L\left(X_{n}^{0}\right)<1.2$. Очевидно, при таком выборе $\left\{Y_{n}^{0}\right\}$ условие (8) выполняется с $K=1$; в силу (11) $P_{n}<1.2 C_{1} / n^{1 / 2} \rightarrow 0$ при $n \rightarrow \infty$, в то же время из (9) следует, что $Q_{n}>0.68 C_{0} R_{3}$.

В заключение автор выражает признательность Ю. В. Прохорову за поддержку и В. Ю. Королеву за полезнье замечания и обсуждения.

\section{СПИСОК ЛИТЕРАТУРЫ}

1. Феллер В. Введение в теорию вероятностей и ее приложения. Т. 1. М.: Мир, 1984.

2. Королев B. Ю., Селивамова Д. О. Несколько замечаний о точности нормальной аппроксимации для распределений случайных сумм. - Вестник Московского унта, сер. вычислит. матем. и кибернет., 1991, № 2, с. 46-53.

3. Круглов $B$. М., Королев $B$. Ю. Предельные теоремы для случайных сумм. М.: МГУ, 1990.

4. Englund G. A remainder term estimate in the random sum central limit theorem. Теория вероятн. и ее примен., 1983 , т. 28 , в. 1 , с. 143-149.

5. Ротарь Г. В. Об одной задаче управления резервами. - Экономика и матем. методы, 1976 , т. 12 , в. 4 , с. $733-739$.

6. Bening V. E., Korolev V. Yu., Shorgin S. Ya. On approximations to generalized Poisson distributions. - J. Math. Sciences. Proceedings of the XVII Seminar on Stability Problems for Stochastic Models, 1996 (to appear).

7. ШІиганов.И. С. Об уточнении верхней константы в остаточном члене центральной предельной теоремы. - В сб.: Проблемы устойчивости стохастических моделей. Труды семинара. М.: ВНИИСИ, 1982, с. 109-115.

Поступила в редакцию 2.IV.1996 